\title{
Idarucizumab hebt die Wirkung des direkten Thrombininhibitors rasch auf
}

Fragestellung: Wie gut wirkt Idarucizumab bei Patienten, die mit Dabigatran behandelt werden und eine schwerwiegende Blutung erleiden oder notfallmäßig operiert werden müssen?

Hintergrund: Dabigatran ist seit mehreren Jahren zur Schlaganfallprävention bei Patienten mit Vorhofflimmern sowie zur Therapie und Prävention tiefer Beinvenenthrombosen zugelassen. Wie bei allen Antikoagulanzien besteht ein Blutungsrisiko und Notfalloperationen sind bei antikoagulierten Patienten grundsätzlich ein Problem. Aus diesem Grund wurde Idarucizumab entwickelt, ein monoklonaler humanisierter Antikörper, der nach intravenöser Gabe mit hoher Potenz Dabigatran bindet und neutralisiert. In der REVERSE-AD-Studie sollte die Wirksamkeit und Sicherheit von Idarucizumab untersucht werden.

Patienten und Methodik: Es handelte sich um eine offene Studie mit Patienten, die unter einer Dabigatran-Therapie eine schwerwiegende, nicht beherrschbare Blutung erlitten, notfallmäßig operiert werden muss-

Pollack CV Jr, Reilly PA, van Ryn J et al. Idarucizumab for Dabigatran reversal - full cohort analysis. N Engl J Med 2017; 377: $431-41$ ten oder einen invasiven Eingriff benötigten, und daher mit $5 \mathrm{~g}$ Idarucizumab i.v. behandelt wurden. Der primäre Endpunkt war die Veränderung des Gerinnungspara- meters der dilutierten Thrombinzeit und der Ecarin-Zeit. Sekundäre Endpunkte waren die Wiederherstellung der Hämostase und Sicherheitsparameter.

Ergebnisse: Insgesamt wurden 503 Patienten eingeschlossen. Davon wiesen 301 Patienten eine schwerwiegende Blutungskomplikation auf und 202 benötigten eine dringende Operation oder invasive Intervention. Bei allen Patienten war die dilutierte Thrombinzeit nach der Gabe von Idarucizumab normalisiert. In der Gruppe mit schwerwiegenden Blutungskomplikationen hatten 137 Patienten eine gastrointestinale und 98 eine intrakranielle Blutung. Bei Patienten mit gastrointestinalen Blutungen kam es nach einer mittleren Zeit von 2,5 Stunden zu einem Stillstand der Blutung. Bei den Patienten mit intrakraniellen Blutungen betrug die Sterblichkeit 16\%. Bei den Patienten, bei denen eine Operation oder eine Intervention notwendig war, wurde diese innerhalb von 1,6 Stunden nach Gabe des Gegenmittels durchgeführt. Bei $93 \%$ der Patienten wurde die periprozedurale oder intraoperative Hämostase vom Operateur als normal angegeben. Schwerwiegende Nebenwirkungen wurden nicht beobachtet.

Schlussfolgerungen: Für Patienten, die unter Dabigatran eine schwerwiegende Blutungskomplikation erleiden oder sich einem dringenden chirurgischen oder interventionellen Eingriff unterziehen müssen, steht mit Idarucizumab ein wirksames Gegenmittel zur Verfügung.

\section{Antikoagulation mit Dabigatran wird sicherer durch Idarucizumab}

Die REVERSE-AD-Studie zeigt sehr überzeugend, dass bei fast allen Patienten, die unter Dabigatran eine Komplikation erleiden oder operiert werden müssen, Idarucizumab innerhalb kürzester Zeit die Wirksamkeit von Dabigatran aufhebt. Für Neurologen ist besonders erwähnenswert, dass die Mortalität von intrakraniellen Blutungen in der REVERSE-AD-Studie nur $16 \%$ betrug, während sie in der RE-LY-Studie [1], als Idaruci-

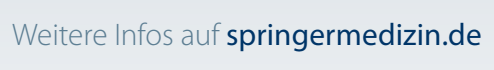

Richtige Therapie des akuten Schlaganfalls zu jeder Zeit und an jedem Ort

Dieser Artikel stellt aktuelle Organisationskonzepte in der Behandlung des akuten Schlaganfalls vor. Sie finden ihn, indem Sie den Titel in die Suche eingeben. zumab noch nicht zur Verfügung stand, zwischen $40 \%$ und $46 \%$ lag. Wichtig ist allerdings auch, dass Idarucizumab nur spezifisch für Dabigatran ist, und bei Patienten, die mit FaktorXa-Hemmern behandelt werden, nicht wirksam ist. Darüber hinaus kann Idarucizumab natürlich nicht Blutungskomplikationen, die durch die zusätzliche Gabe von Thrombozytenfunktionshemmern bedingt sind, antagonisieren. Die Gabe von Idarucizumab entbindet auch nicht von allen anderen Maßnahmen zur Blutstillung, wie beispielsweise der Kompression der Blutungsstelle oder einen endoskopischen Eingriff zu bevorzugen. In der REVERSE-AD-Studie wurden keine Patienten mit Idarucizumab behandelt, die einen ischämischen Insult erlitten hatten und Kandidaten für eine Thrombolyse waren. Dies wird jedoch in der Zwischenzeit in Deutschland routinemäßig so gehandhabt [2].

\footnotetext{
Referenzen

1. Connolly SJ et al. N Engl J Med 2009; 361: 1139-51

2. Kermer $P$ et al. Int J Stroke 2017; 12: 383 - 91
} 